\title{
Death and Dying: A Nursing Focus on the Care of the Patient
}

\author{
Kerry Brodbeck, Nancy Duphily* \\ Department of Nursing, Fitchburg State University, USA
}

Copyright $@ 2019$ by authors, all rights reserved. Authors agree that this article remains permanently open access under the terms of the Creative Commons Attribution License 4.0 International License

\begin{abstract}
The role of the nurse is often associated with healing and helping bring people back to health. While this is a large aspect of the nursing profession, the nurse also cares for patients who are dying. The care delivered at this time revolves around the comfort of the patient, as well as ensuring that all of the patient's needs are met, including any cultural or spiritual needs. In order for the care of the patient to transition from curative to comfort measures, it is necessary for a diagnosis of dying to be made. This diagnosis should then be communicated with the patient and family. When the diagnosis of death is not communicated in a timely manner, it has been found that the patient's do not receive quality end of life care. This diagnosis surpasses all other diagnoses and shifts the focus towards the comfort and well-being of the patient. Care at the end of life includes pain management, discontinuation of routine vital signs and laboratory tests and coordinating care with other professionals to ensure the best quality care is being offered. Since the end of life is a new experience for most, it is important that the nurse informs the patient and the family of interventions that will occur and all resources available to them. Palliative care is a resource that can be implemented along curative measures at any stage of a disease, which assists the patient with pain and symptom management. As the disease course continues and the patient's life expectancy is less than six months, hospice care is a resource that should be offered. The nurse, along with the rest of the care team, should ensure that these resources, along with legal documents such as advanced directives or healthcare proxy forms are available to the patient. At the end of life, the nurse is on the front lines to ensure that the patient is receiving the best quality care possible. This is done by communicating and listening to the patient and developing a therapeutic relationship with them.
\end{abstract}

Keywords Death, Dying, Hospice and Palliative Care, Bereavement, Healthcare Proxy, Culture

\section{Modern Society's View on Death and Dying}

There is one thing in life that is absolutely certain: every individual who is born will one day die. Even though this is the only certainty in life individuals' face, the concept of death and dying is often approached with feelings of fear, anxiety, and reservation. These fears are often in regards to pain, suffering and the unknown. Thoughts in relation to death and dying have shifted throughout recent history, with many believing that modern medicine and a changing society have contributed to an increase in anxiety regarding this topic.

One theory for an increase in fear and anxiety towards death is the advancements made in modern medicine [1]. Until the $19^{\text {th }}$ century, people were faced with death frequently throughout their lives, and most people died at home. The caregivers were typically family members and loved ones. However, in today's society, death has become far more institutionalized, and the majority of deaths occur within the hospital or at a long-term care facility. Medical practices have distanced the dying from the rest of the living. This has resulted in a society that is not exposed to death until it affects oneself or an immediate family member. In the United States, our medical advancements such as vaccinations, modern technology, and surgical interventions contribute to a longer lifespan and fewer fatalities than that of the $19^{\text {th }}$ century [2].

The permanence and complete unpredictability of death is a focal point for anxiety for many individuals in today's society. It is common practice to deny our own mortality, believing that death can happen to our neighbor, but never to our loved ones or ourselves. This thought process often results in individuals testing their own mortality by partaking in risky behaviors, such as speeding on the highway, drinking and driving, or cigarette smoking. These behaviors, which disregard our own mortality, can result in a total lack of preparation for when death does actually occur. Roughly, one quarter of adults have given little, if any, thought on how they would like doctors or medical 
professionals to handle their medical treatment at the end of their lives. This often results in extended pain and suffering for those individuals during death and dying [3].

In many cultures and religions, death is thought to be a reward for a life of suffering. For example, Hyolmo Buddhists in Nepal believe that death is an intricate art that needs to be learned throughout life [4]. The decline of mainstream religion in the United States may be a contributing factor for the rise of anxiety and fear regarding death and dying. The presence of mainstream religion allowed society as a whole to feel that there may be a purpose after death, such as reuniting with family members, reincarnation, or being righteous enough to view God. In the last ten years, the percentage of Americans who believe in God, pray daily and regularly gone to church has declined [5]. This trend, while not indicating a decline in a belief of the afterlife, shows that the nation as a whole is not partaking in religious activities as often, which can correlate to an increase in fear and anxiety regarding death and dying.

The American population is growing older and living longer than ever before. It is estimated that the 85 and older population will triple in size from 5.4 million to 18 million by 2050 [6]. This population is also expected to live with several comorbidities and chronic diseases, which in turn will result in more care needed at the end of life. In order for this population to receive the best possible care, it is necessary that healthcare workers, especially nurses, are able to approach the death and dying process and patient appropriately. This stems from proper education on death and dying, open communication with the individual and their loved ones, and collaboration with other healthcare professionals.

\section{Diagnosing Dying}

The first step in ensuring that the patient at end of his or her life is receiving quality care, involves acknowledging that the patient is dying. In order for a patient to be diagnosed as dying, it is necessary that the multi-professional team involved in the patient's care all agree that death is probable [7]. If this is not found, the patient and family may be unaware that death is imminent. This can result in a patient receiving conflicting information, a patient dying with uncontrollable symptoms, and the cultural, spiritual or legal needs of the patient left unconsidered [7].

People die of many different reasons and causes. The majority of deaths that require long-term care at the end of life are cancer, congestive heart failure, chronic obstructive pulmonary disorder (COPD), dementia, and stroke. Signs of imminent death vary for these conditions. In patients with cancer, the dying phase is often associated with the patient becoming bedbound, only able to take sips of fluid, no longer able to take oral drugs, and in a semi-comatose state. However, in patients with Congestive Heart Failure, the evidence of dying includes contain a feeling of anxiety, loss of appetite, weakness, fatigue and shortness of breath at rest, and swelling of the face, limbs and abdomen [8]. It is necessary that the care team, including doctors, nurses and case managers, are aware of these signs and symptoms. It is equally important that when this is realized, the patient and family are informed.

It is often found that physicians and nurses are able to recognize imminent death in their patients; however, that information is not always relayed to the patient and their family. A study by Sullivan, Lakoma, Matsuyama, Rosenblatt, et. al, (2007), highlights the high percentage of physicians who are aware of imminent death in their patients, and the contrasting percentage of patients and families who are informed of this finding. The study found that a portion of physicians (38\%) were able to identify imminent death during their patient's admission; however, a majority (86\%) were able to identify imminent death at some point during the hospitalization. Only half of the patients were actually told that death was imminent, and the majority of those were lucid patients, younger patients, and patients where one member of the care team was certain that they would die [7]. This, in turn, results in a significant population of patients who do not receive quality end of life care. This study showcases the healthcare team's awareness of a patient's imminent death, and the lack of communication with patients and families at this time.

Although the diagnosis of dying can seem like the healthcare team is losing hope, it is necessary in order for the patient to receive the best quality care. This diagnosis creates a certain perspective needed by the patient, family and care team. In the inpatient setting, this diagnosis would limit the amount of invasive procedures being ordered, the frequency of vital signs being taken, and the need for telemetry, glucose, or intake monitoring. It would then create an environment where the patient's comfort and emotional needs are the foreground for care. This would include more frequent assessments by the nurse for pain, orders for hospice consultation, and comfort measures such as oxygen or a Foley catheter. The diagnosis of dying is necessary because it trumps all other diagnoses, and the needs of the patient will be in the foreground of care [8].

\section{The Care of the Dying Patient}

The role of the nurse extends into many realms of the healthcare setting. During the end of life, nurses are present for bedside care, members of the consultation team, and case managers. End of life care can occur in in-patient acute care settings, residential hospices, skilled-nursing facilities, and the patient's home. During this time, the registered nurse has a changed mindset from interventions and treatments to one of comfort, support and symptom 
management [9].

In order to provide the best quality care for patients at the end of life, the registered nurse must be able to confront the possibility of death and be comfortable communicating with the patient and family. This is a skill that is developed and refined through experience, and for the novice nurse, it is often one of the most difficult conversations to have with patients and loved ones. However, through experience, the nurse learns the best methods and tactics to have a therapeutic conversation. In recent years, nursing education systems have begun to highlight the importance of end of life care and communication in their curriculum [9]. The sooner nurses are exposed to these topics and conversations; the sooner nurses become effective therapeutic communicators at the end of life.

Communication at the end of life is vital to quality care. The patient and family needs to fully understand the current situation and the likelihood that death is approaching. If this is not established, the patient and family may still be hoping for a cure, where the mindset should be on comfort measures and the patient's wishes. Once the current situation is fully understood, it is necessary that the nurse and other members of the care team have a conversation with the patient about what they envision for their death. Some people are adamant about dying in their home, and if possible, it can be arranged for the individual to be discharged home with hospice services. Some others wish to speak to a priest, rabbi, chaplain, or other spiritual person of their choice. When an open line of communication is established, the majority of the patient's wishes at the end of life can be met. This results in a higher degree of trust between the patient and the nurse, which in turn results in better quality care [7].

The end of life brings many changes for the patient and their family. They may be undergoing a change in familial or occupational roles, financial hardship, or even the loss of human worth. At this time, compassion and kindness can help patients feel like they are not alone in this changing time. The nurse exhibits compassion by staying with a patient and answering questions, or even sitting with them in silence when they no longer have anything to say but do not want to be alone. Conversations about the patients' past life experiences often allow them to feel as if their life has had worth and meaning, and in turn may result in less resentment about their death. It often means the nurse has to go the extra mile beyond what the doctor ordered or which medications need to be administered; however, it allows the patient and the nurse to build a relationship that will provide both of them healing. When the nurse is kind and compassionate, the patient will feel as if they are not facing this unknown by themselves [10].

Caring for a patient at the end of life can take a toll on the nurse as well. Nurses are compassionate, considerate, and genuine. When quality care by the nurse is delivered, the conversations and experiences result in real relationships between the nurse and the patient. When the patient dies, it is normal for the nurse to feel similar emotions experienced by the family and loved ones. At times, seeing the emotion felt by the nursing staff can be therapeutic to the family, as it symbolizes the valued life that all cherished. It is important, however, that nurses understand that their job is not complete when the patient dies. They also must be able to therapeutically communicate and help the bereaved family and loved ones [11].

At the end of life, it is important that the patient and family are aware of the physical changes that may occur. When death is near, it is normal for the patient to sleep more, or seem to be in a semi-comatose state. Due to this state, it is important that the nurse and family create a relaxing environment. This can be done by playing the patient's favorite music or limiting the amount of visitors when the patient is resting.

Another change that often occurs is that the patient eats less and does not drink as much water. This can result in an extremely dry mouth for the patient, which is very uncomfortable. Mouth care by the nurse should be completed per facility protocol, however, ice chips, ointments and mouth sponges should be included and offered as needed. As death approaches, the patient may also become incontinent. It is important that the family and patient, if appropriate, are educated that this is a normal occurrence at this time. The care-team, including physicians and nurses, will decide if the insertion of a Foley-catheter is appropriate. In the case of urinary retention, often a catheter will be inserted to drain the bladder and help restore patient comfort

Nursing care at the end of life is based on providing quality and competent care for the patient that is centered on their comfort. This often includes discontinuing any medications and tests that are unnecessary and may be uncomfortable for the patient, including routine vital signs, laboratory draws, or frequent oral medications. Providers would have orders for pain management to be delivered to the patient via intravenous routes as needed. In many cases, patients near the end of death may not be able to verbalize their pain or their symptoms. It is important for the nurse to understand common signs of pain, such as grimacing, moaning, or being restless. It is often said that hearing is the last sense to leave us, and the same is true for the semi-comatose patient at the end of life. It is important that the nurse and family communicate to the patient when anything such as medication administration, turning or repositioning is being done. This helps keep the patient calm and oriented for as long as possible.

\section{Resources Available to the Dying Patient}

The end of life is a new and daunting experience for which most are not fully prepared for. It is important that the patient and the family receive guidance on not only 
how to provide the best care, but also on how to manage legal documents and resources that may be available to them. It is important that information about these recourses are provided to the patient or family as early as possible, as it will help them feel more in control and prepared for the end of life [12].

When patients are diagnosed with a terminal or serious illness, palliative care should be implemented alongside curative measures. When palliative care is provided early on in the disease process, it can help the patient address their needs head on, as well as help the patient and their families acknowledge their priorities. Palliative care focuses on the comfort and well-being of the patient, and ensures that all of the patient's needs are being met. The majority of palliative care is done by the nurse themselves, however, the physician completes orders and assessments as well. The main palliative tasks done by the nurse include pain management, family and patient counseling and educating, medication administration and giving emotional and physical comfort [13].

All nurses in all settings can practice palliative care. The American Nurses Association and The Hospice and Palliative Care Associated made a call for action in 2017 for all nurses to lead and transform the current Palliative Care program. They developed an online education unit, the "End of Life Nursing Education Consortium", to help all nurses feel capable of providing quality palliative care to their patients. There are also nurses who specialize in palliative care. They have an expert knowledge on the pathophysiology of diseases, an advanced assessment on symptom and pain management, and collaborate with the unit nurse to provide the best care and planning for the patient. Palliative care can also be delivered to the community via home nursing visits. These visits limit the cost to the patient, reduce the frequency of in-patient hospital stays, and help coordinate care between the nurses, physicians and family members [13].

As the course of the disease continues and the physician rules that the life expectancy of the patient is less than six months, a patient and their family may elect to begin hospice care. At this point, curative measures are no longer pursued, and the main focus is of symptom management and comfort. The hospice care team may include a physician, nurse, a hospice aid, chaplain or a social worker if needed, and the care can be conducted in many different locations. Often, people elect to have hospice care in their own home or in a residential house often called a "hospice house" [14]. This provides the privacy and intimacy between the patient and their family that may not be feasible in the inpatient setting.

Patients' wishes regarding their medical treatments and wishes at the end of life need to be legally documented. A document such as the Advanced Directive allows the patient to document their wishes regarding the medical treatments at the end of life. In some cases, people are not able to communicate their wishes when they are near death.
This form is a legal document on how they would want to be cared for, only in the case where they are unable to communicate it themselves. An advanced directive can be edited over time to account for a change in wishes or health status. Components of the advanced directives include decisions of whether a patient wants to be put on a ventilator, if artificial nutrition is to be implemented, or if any comfort measures should be added or omitted. Before the advanced directive can guide decision-making, two doctors need to be in agreement that the patient is unable to make their own medical decisions. This form is not only for the sick and dying, it is recommended to all because a medical emergency can arise at any time.

Another legal form that is important at any point in a hospitalization, particularly at the end of life, is the Healthcare Proxy form. This form allows the patient to appoint an individual they trust to be their healthcare agent. In the case that the patient is unable to communicate, the healthcare proxy will make the decisions on their behalf. It is important that before naming a healthcare proxy, the patient discusses their wishes to them, and in turn, it is important that the proxy acts upon those wishes and not their own. In order for the best care to be delivered at the end of life, the patient should have these legal documents in place; however, it is the job of the nurse or case manager to ensure that the patient is aware of these documents and the importance of them.

At the end of life, it is important to have an open discussion with the patient regarding their spiritual needs. This is something that needs to be discussed directly with the patient or a family member, and assumptions should not be made based on race, ethnicity, or cultural norms. An assessment regarding spirituality should also be done at the end of life to see if there is a loss of faith or hope that can lead to anxiety, depression, isolation or a difficult time dealing with symptoms. If this is not addressed, the nurse may not be able to provide the best care the patient needs. In the inpatient setting, hospital chaplains are available. Chaplains work with local religious or spiritual leaders who will visit and provide support for patients who are at the end of life, or in any stage during a hospitalization. It is also important that in other settings, such as the home, the patient is given the important resources regarding spiritual care as well. In many cases, the nurse or family can arrange for the patient to receive the Eucharist, be seen by their pastor, or if they are Catholic, have a Last Rites Ceremony. A spiritual support plan may be developed as well. This includes specific ways to honor the patient's beliefs during treatment, such as not receiving blood products for patients who are Jehovah Witnesses, or scheduling a regular meeting with a spiritual advisor. The nurse must advocate for their patient, and ensure that all needs are being met at the end of life [15].

At the end of life, one of the most reliable resources for the patient is often their family. In most cases, the family provides roughly $80 \%$ of elder care, including lifting, 
bathing and meal delivery. The end of life is a changing time in the structure of a family, and often family functioning, communication, and dynamics are impacted. Families tend to need emotional support during this time, which often includes being well informed. When the family is informed that the end of life for their loved one is near, it allows them to have more time to process and be prepared for what is to come. It also allows the family to feel less regret regarding the passing of their loved one. The nurse helps provide this care by explaining all interventions that are being conducted, as well as what they should expect to see as death approaches. The nurse also should help manage family dynamics. At the end of life, family-conferences are an intervention that can be held routinely. It allows the staff to assess the functioning within the family, while keeping the family informed as to what is occurring [11]. It is important that the care of the family extends after the death of the patient. The bereavement process does not end when the family leaves the hospital, and offering to be a resource to the family after the care of the patient is completed, can help the grieving process.

\section{Delivering Culturally Sensitive Care}

As a nurse, it is extremely important that culturally sensitive care is practiced. This includes delivering care that is tailored to the spiritual or religious beliefs of the patient, modesty issues, the patient's perception of their illness, and their dietary needs. It is impossible for the nurse to be aware of the specifics of all cultures, but care should be sensitive towards all cultures. In order for this to be possible, the nurse must ask the patient to identify their wishes and beliefs. Some considerations to make when gathering information include the following: family involvement in patient care, spiritual or religious beliefs that impact the health interventions of the patient, religious or spiritual leaders who should be contacted, and who can be present for the death [16].

Some cultures, including Eastern Asian cultures, believe it is inappropriate to openly discuss death, and that bad things occur when they are spoken out loud. Also, the patient themselves is often not informed of all the happenings, but the family are usually well aware. This can lead to issues with pain management, which is a significant aspect of care at the end of life. When the topic of death cannot be discussed, it is difficult for the care team to initiate the conversation about transitioning to comfort measures and pain managements, which in turn can lead to a patient dying with uncontrolled symptoms [17].

For some cultures, the decisions regarding the end of life are not made by the patient themselves, but by the family as a whole. Western cultures typically value the individual's independence and autonomy in making their own decisions when they are able to. Many Eastern cultures, however, value making these types of decisions as a family. In many
Asian cultures, as well as Mexican American and Black American cultures, there is a believed duty by the family to care and make decisions for their sick or elderly loved ones [17].

Another difficulty that may arise when caring for patients of a different culture is a language barrier. It is important that when translating between the nurse and the patient, that official interpreter services are used. The use of the family is not appropriate. When the family is used, the nurse cannot be certain that all medical jargon is being relayed to the individual, or if all information is relayed to the patient. Interpreter services are offered by most facilities, and they should be utilized when caring for a patient when there is a language barrier [18].

Cultural beliefs extend past just pain management and family participation in care. It is important that the nurse asks about certain restrictions the patient may follow, such as dietary restrictions, limit of analgesics, or the administration of blood products. At the end of life, Muslims may refuse the use of analgesics to remain pure for when they meet Allah. This varies among the individuals, but individuals who are more traditional believe that they need to have a clear head and be mentally alert. Many cultures have dietary restrictions and limitations. Muslims often avoid pork and practice fasting during the month of Ramadan or other religious days. Fasting is also common in many South Pacific cultures when they feel as if they are nearing death. Hindus are often vegetarian and depending on their orthodoxy, the Jewish culture often follows a Kosher diet. While caring for the individual at the end of life, it is important that the nurse considers the culturally beliefs of the patient [15].

\section{Conclusions}

The death and dying process is daunting and filled with the unknown for most patients. Advancements in modern medicine have separated the death from the rest of the living, and we often are not exposed to death until it affects ourselves or a close family member. Due to this situation, it is important that the diagnosis of death be relayed to the patient in a timely manner. This gives the patient, their families, and the care team time to shift their focus from curative measures and interventions, to maintaining the patient's comfort. This care can occur in many settings, including the patient's home, a long-term skilled nursing facility, inpatient acute care or hospice homes. It is important that in all of the settings, the nurse is able to communicate comfortably with the patient and loved ones about the dying process. This skill is developed over time and may be difficult for the novice nurse; however, open communication with the patient and family helps aid that the patient is comfortable and all needs are being addresses. This includes all physical needs, but also asking if any spiritual or cultural considerations should be included, such 
as meeting with a spiritual leader or certain care given to the body after death. The nurse also coordinates care with other members of the care team, including case managers and social workers. This team ensures that the patient is aware of all resources that are available to them, including palliative and hospice care, legal documents, and family care. The nurse utilizes their team mentality to provide the patient with the best quality care possible. At the end of life, the nurse is a valuable resource in providing patients and loved ones with support, education and care.

\section{REFERENCES}

[1] Field, M. J., \& Cassel, C. K. (1997). Approaching death: Improving care at the end of life. Washington, D.C.: National Academy Press

[2] Kübler-Ross, E. (1969). On Death and Dying. New York City, New York: Macmillan

[3] Views on End-of-Life Medical Treatment. (2013). Pew Research Center: Religion \& Public Life. Retrieved from: http://www.pewforum.org/2013/11/21/views-on-end-of-lif e-medical-treatments/

[4] Desjarlais, R. (2016). Subject to Death: Life and Loss in a Buddhist World. Chicago, Illinois: University of Chicago Press

[5] U.S. Public Becoming Less Religious (2015, November 3). Pew Research Center: Religion \& Public Life. Retrieved from: http://www.pewforum.org/2015/11/03/u-s-public-be coming-less-religious/

[6] Ortman, J. M., Velkoff, V. A \& Hogan, H. (2014). An aging nation: The older population in the United States: Population estimates and projections. United States Census Bureau

[7] Sullivan, A. M., Lakoma, M. D., Matsuyama, R. K., Rosenblatt, L., Arnold, R. M., \& Block, S. D. (2007). Diagnosing and discussing imminent death in the hospital: A secondary analysis of physician interviews. Journal of Palliative Medicine, 10(4), 882-893. doi:10.1089/jpm.2007.0189

[8] Ellershaw, J., \& Ward, C. (2003). Care of the dying patient: The last hours or days of life. BMJ (Clinical research ed.), 326(7379), 30-4

[9] Schroeder, K., \& Lorenz, K. (2018). Nursing and the future of palliative care. Asia-Pacific journal of oncology nursing, $5(1), 4-8$

[10] Rousseau, P. (2001). Kindness and the end of life. The Western journal of medicine, 174(4), 292

[11] Seaman, J. B., Bear, T. M., Documet, P. I., Sereika, S. M., \& Albert, S. M. (2014). Hospice and family involvement with end-of-life care: Results from a population-based survey. The American journal of hospice \& palliative care, 33(2), 130-5

[12] Zaider, T., \& Kissane, D. (2009). The assessment and management of family distress during palliative care. Current Opinion in Supportive and Palliative Care, 3(1), 67-71. http://doi.org/10.1097/SPC.0b013e328325a5ab

[13] Pesut, B. \& Greig, M. (2018). Resources for educating, training, and mentoring nurses and unregulated nursing care providers in palliative care: A review and expert consultation. Journal of Palliative Medicine, 21(S1) https://doi.org/10.1089/jpm.2017.0395

[14] Hospice Homes, (2015). National Hospice and Palliative Care Organization

[15] Feser, L. (2003). Cultural Competence and cross cultural care at the end of life. Virtual Hospice (1)14

[16] Saccomano S, Abbatiello G. (2014). Cultural Considerations at the end of life. The Nurse Practitioner; 39(2), 24-31.

[17] Martin, E. M. and Barkley T. W., (2016). Improving cultural competence in end-of-life pain management. Nursing2016 (46) 1 